\title{
In Situ Wood Fiber Dyeing Through Laccase Catalysis for Fiberboard Production
}

\author{
Alberto Colella ${ }^{1}$, Addolorata De Chiaro ${ }^{1}$ and Vincenzo Lettera ${ }^{1,2 *}$ \\ ${ }^{1}$ Biopox srl, Viale Maria Bakunin, Napoli, Italy, ${ }^{2}$ Department of Chemical Science, University of Naples Federico II, Napoli, Italy
}

OPEN ACCESS

Edited by:

Abdelmageed M. Othman,

National Research Centre, Egypt

Reviewed by:

Markus Euring,

Wood Technology and Wood Based

Panels, Germany

Jolanta Polak,

Maria Curie-Skłodowska University,

Poland

Andrea Scozzafava, University of Florence, Italy

Marilize Le Roes-Hill,

Cape Peninsula University of

Technology, South Africa

*Correspondence:

Vincenzo Lettera lettera@biopox.com

Specialty section:

This article was submitted to Industrial Biotechnology,

a section of the journal

Frontiers in Bioengineering and

Biotechnology

Received: 17 September 2021

Accepted: 26 October 2021

Published: 03 December 2021

Citation:

Colella A, De Chiaro A and Lettera V (2021) In Situ Wood Fiber Dyeing

Through Laccase Catalysis for

Fiberboard Production.

Front. Bioeng. Biotechnol. 9:778971.

doi: 10.3389/fbioe.2021.778971
The aim of the present work was to develop an innovative and environmentally friendly process for wood fiber dyeing and to produce 3-dimensionally fully colored medium-density fiberboard (MDF). The potential of laccase-catalyzed polymerization of selected precursors to form dyes useful in fiberboard manufacturing, a technique used for the first time in this field, was demonstrated. Some of the 7 aromatic compounds tested yielded colored products after laccase treatment under both acid and alkaline conditions, and a good variety of colors was attained by using mixtures of two different monomers. To demonstrate the coloration and design potential of laccase conversion of aromatic compounds, MDFs were enzymatically dyed using an in situ one-step laccase-catalyzed coloration process, and the results were compared against commercial MDFs obtained by using organic coloring agents. Important advantages over conventional processing methods include good color fastness and, in some cases, new hydrophobic properties, allowing designers and woodworkers to explore the beauty of textures and the use of simpler and milder processing conditions that eliminate harsh chemical use and reduce energy consumption.

Keywords: in situ dyeing, medium-density fiberboard, POXA1b, enzyme oxidation, colors

\section{INTRODUCTION}

In the frame of circular economy, wood wastes are becoming increasingly attractive to meet the enormous demand of wood that exists in a wide range of industries (Euring et al., 2011). When trees are processed, wood chips and sawdust are produced, in addition to thinning and residual wood. These residues can be used as raw material for furniture and interior design in the production of wood-based devices, such as wood medium-density fiberboard (MDF). Wood fibers are generally blended with resin, wax, and other additives; dried; and pressed. After pressing, the boards are cooled, sanded, trimmed, and sawed to final dimensions. MDFs may also be painted or laminated; however, the coloring procedure generally covers only the small part of wood fibers visible on the surface.

At the end of the last century, Valbopan S.A. (Leiria, Portugal) presented a special wood-based fiberboard with a "wooden natural" surface and with color throughout the thickness to the market. Manufacturers, architects, and technicians have demonstrated technical, aesthetic benefits, and advantages of its use. The final product is a panel made of wood fibers colored during the production process. Although this technology results in an innovation in the dyeing process, that is, third dimensional dyeing, the fibers still need to be soaked with organic colorants and bounded to one another through chemical agents, which usually result to be toxic or recalcitrant.

The potential for the use of wood wastes as ecofriendly, sustainable, recyclable, and reusable product is not fully exploited yet because this requires new processes to be set up along the entire 
value-added chain, from the adhesive used during fabrication to the chemical components used in the dyeing/laminating process. As a fact, the trend in the global market has shifted toward the use of fiberboards not containing toxic, hazardous, or oil-derived chemicals (Euring et al., 2011). In this perspective, the forest industry is increasingly adopting enzyme-based technologies for binderless or self-bonding fiberboard manufacturing (Vitronea et al., 2021).

Wood-decay or parasitic fungi are generally able to digest wood; thus, their enzymes have stimulated several studies on the exploitability of fungal enzymes in the forest industry (López et al., 2017; Andlar et al., 2018; Zhuo and Fan, 2021). Among them, laccases (benzenediol: oxygen oxidoreductase; EC 1.10.3.2) have been extensively characterized because of their potential for industrial applications. These enzymes, belonging to the multicopper oxidase family, catalyze the monoelectronic oxidation of a broad range of substrates, such as phenols and aromatic or aliphatic amines, to their corresponding radicals, using molecular oxygen. Laccases can oxidize aromatic polymers, such as lignin, through free radical reactions (Sun et al., 2013). This catalytic activity has been applied to activate lignin to produce lignin-based adhesives and to attain physically and/or chemically modified wood fibers (Rowell, 1996; Hu et al., 2016; Agustin et al., 2021).

Most of the laccase-based approaches, related to wood fiber treatment, have been focused on the improvement of MDF mechanical properties through the augmentation of bonding strength within and between the fibers (Nasir et al., 2014; Nasir et al., 2016). Surprisingly, there is a complete lack of data concerning laccase applications in the wood dyeing sector. As a fact, laccases are able to catalyze coupling of aromatic compounds, such as phenols and diamines, producing colored oligomers or polymers in the presence of different solid porous matrixes (Su et al., 2018). Several research groups have already applied in situ enzymatic synthesis of dyes in existing industrial products such as cotton, wool, and hair dye (Kim et al., 2007; Jeon et al., 2010; Pezzella et al., 2016; Sousa et al., 2016; Kumar et al., 2018; Prajapati et al., 2018; Yuan et al., 2018).

In our opinion, eco-compatibility and catalyzing properties of laccases could be applied to the in situ dyeing of wood fibers. MDF dyeing works by penetrating the wood fibers, soaking in and drying in the wood pores. In order to successfully and permanently dye MDFs, dyes have to be applied before the panel is sealed by polyurethane or other finishing agents.

The aim of this research was to develop a laccase-catalyzed in situ dyeing process for wood fibers that could be integrated into the already existing industrial processes, as an alternative to conventional MDF dyeing methods. Several studies, exploring laccase catalysis, suggest that a wide range of color diversity may be achieved through a combination of different precursors incubated together in the presence of enzymes (Kim et al., 2007; Jeon et al., 2010; Pezzella et al., 2016; Kumar et al., 2018; Prajapati et al., 2018; Yuan et al., 2018). This study was focused on investigating the dyeing reactions using laccase catalysis on seven selected substrates combined in heteromolecular mixtures that led to formation of mixtures of heteropolymers or homopolymers. Color parameters of heteromolecular mixture reactions were compared with those of single monomer reactions, by also considering reaction processing parameters: acid and alkaline $\mathrm{pH}$ and reaction times. Furthermore, wood fiber wettability was tested to determine the related functionalization degree, due to the modification of their chemical surface properties. To date, this is the first reported application of laccases in wood dyeing.

\section{MATERIALS AND METHODS}

\section{Materials}

Recombinant POXA1b laccase from Pleurotus ostreatus, expressed in the yeast Pichia pastoris under the control of the AOX1 promoter, was produced by BioPox srl. Bleached pine wood fibers were provided by Wetlands Engineering (Belgium). The wood fibers were stored at $22 \pm 3^{\circ} \mathrm{C}$ in a hermetically sealed plastic bag under dry conditions.

Valchromat ${ }^{\circledast}$ commercial fiberboard samples were provided by a local joinery. As reported in the related datasheet, the fiberboards were colored during the production process with organic coloring agents and chemically bonded by a melamine-urea-formaldehyde resin. All reagents were purchased from Sigma-Aldrich Corp. (St. Louis, MO).

\section{Enzyme Activity Measurement}

Laccase activity was determined using 2,2'-azino-bis (3ethylbenzothiazoline-6-sulfonate) (ABTS) as a substrate following the reaction at $420 \mathrm{~nm}\left(\varepsilon_{420}=36 \times 10^{3} \mathrm{M}^{-1} \mathrm{~cm}^{-1}\right)$, as previously reported (Lettera et al., 2010).

\section{Dye Synthesis}

The following chemicals were used as precursors for the dye synthesis: resorcinol, $p$-phenylenediamine, $m$-aminophenol, 4,5-diamino-1-(2-hydroxyethyl)pyrazole sulfate, 2,4,5,6tetraaminopyrimidine sulfate, syringic acid, and 2,5diaminobenzenesulfonic acid (2,5-DABSA). All precursors were purchased from Sigma-Aldrich Corp. (St. Louis, MO).

The reactions were carried out through incubation of $5 \mathrm{mM}$ of each precursor dissolved in $100 \mathrm{mM}$ sodium citrate at $\mathrm{pH} 3$ or in $50 \mathrm{mM}$ Tris- $\mathrm{HCl}$ at $\mathrm{pH}$ 9. Heteromolecular synthesis was achieved by mixing a couple of precursors in a 1:1 $\mathrm{M}$ ratio. Each solution was incubated with $1 \mathrm{U} / \mathrm{ml}$ of laccase in aqueous solution up to $24 \mathrm{~h}$ at $22 \pm 3^{\circ} \mathrm{C}$ without stirring. The reactions were followed by registering the absorbance spectra (400-800 nm) at the beginning of the reaction and after 1, 5, and $24 \mathrm{~h}$ and subtracting spectra of substrates in the absence of enzyme as blank (UV-1600PC VWR spectrophotometer). All measurements were performed in triplicate, and the standard deviation of the absorbances was evaluated at the $\lambda_{\max }$ for each assay.

\section{In situ Wood Fiber Dyeing}

Wood fibers $(1.5 \mathrm{~g})$ were dyed by soaking the fiber bulk in $50 \mathrm{ml}$ of dyeing solution. Each dyeing bath was formulated by $5 \mathrm{mM}$ of each precursor in homogeneous solution or by $5 \mathrm{mM}$ of each 
precursor in a heteromolecular mixture in both $\mathrm{pH}$ conditions and incubated with laccase. The laccase activity was increased up to $40 \mathrm{U} / \mathrm{ml}$, in order to allow homogeneous dyeing of the fibers and to enhance the low diffusivity of the catalyzer into the solid matrix. The dyeing procedure was carried out for $5 \mathrm{~h}$.

Wood fibers treated with dyeing solutions, which contain precursors in the absence of enzymes or laccases in the absence of oxidizable precursors, were used as control. The treated wood fibers were washed thoroughly with deionized water until the washing solution became colorless. Finally, the wet fibers were dried at $40^{\circ} \mathrm{C}$ for 7 days in the oven.

\section{Form-Pressing Wood Fibers}

$1.5 \mathrm{~g}$ of wet dyed fibers were directly blown into a tube with a cross-sectional area of $9.6 \mathrm{~cm}^{2}$ and constantly pressed using a press (manual hydraulic press $10000 \mathrm{Kg}-10 \mathrm{~T}$ with pressure gauge MA PE PE10, EchoENG, Italy) at $10 \mathrm{KPa}$ for 5 days at $40^{\circ} \mathrm{C}$ to fabricate a mat of uniform thickness. Finally, a form-stable, formpressed dried sample was removed from the press.

\section{Color Values}

The colorimeter Mightex's SLB-1200-1 universal LED driver was used to determine the color values $L^{*}$ (lightness to darkness), $\mathrm{a}^{\star}$ (redness to greenness), and $\mathrm{b}^{\star}$ (yellowness to blueness) of each enzymatically treated and untreated sample, represented by the CIELAB color space system. Each sample of undiluted colored solutions or pressed colored wood fibers was measured three times. An average color measurement was calculated from the data collected for each sample in triplicate. Color values were converted into the RGB system through the conversion tool available online (colorizer.org), in order to graphically represent the variety of colors achieved in liquid solutions. When the RGB colors related to the replicates of each experiment appeared indistinguishable to the human eye, the variability was considered negligible.

\section{Color Fastness to Water}

Color fastness to water of the wood fibers dyed was evaluated by dipping dyed samples in $200 \mathrm{ml}$ of hot water $\left(80^{\circ} \mathrm{C}\right)$ for $2 \mathrm{~h}$, as reported by Zhu and coworkers (Zhu et al., 2018). Afterward, the color values of the treated samples were recorded using a colorimeter. Color differences between two samples were represented as $\Delta \mathrm{E}$ and were calculated using Eq. (1) (Schanda, 2007):

$$
\Delta \mathrm{E}=[(\Delta \mathrm{L} *) 2+(\Delta \mathrm{a} *) 2+(\Delta \mathrm{b} *) 2]^{1 / 2},
$$

where $\Delta \mathrm{L}^{*}, \Delta \mathrm{a}^{*}$, and $\Delta \mathrm{b}^{*}$ represent the differences between the corresponding units of each sample.

Surface color change values of wood fibers before and after dyeing were determined through solid color measurement using the CIELAB color system. The color values were measured in three portions of each sample. The average value of each chromaticity parameter was recorded, and the total color difference $(\Delta \mathrm{E})$ was calculated using Eq. (1).

The total color difference $\Delta \mathrm{E}$, obtained using Eq. (1), was used to measure the color fastness in response to treatment with water.
All measurements of color values were performed in triplicate, and the corresponding standard deviations were evaluated as $\Delta \mathrm{E}$. A difference in colors, which corresponded to a $\Delta \mathrm{E}$ less than 3 , was indistinguishable to the human eye, and the related variability was considered negligible.

\section{Contact Angle and Drop Absorption Time Measurements}

The water contact angle was measured with a deionized water droplet on the surface of the form-pressed dried sample at $22 \pm$ $3^{\circ} \mathrm{C}$. A dosing volume of the droplet was set at $20 \mu \mathrm{L}$ using a Hamilton $500-\mu \mathrm{L}$ syringe. The receiving surface was placed on the stationary drum, and a picture of the drop-receiving surface interaction was taken by using a transversely positioned digital camera (Sony 2.6 pol LCD with built-in flash 20.1 MP) in the auto mode. Measurements were taken $1 \mathrm{~s}$ after the drop release. The images were analyzed by ImageJ $1.45 \mathrm{~s}$ Drop Shape Analysis plugin. Moreover, by measuring the time at which the drop touches the wood fiber surface and the time at which the drop disappears from the surface, we determined the drop absorption time of the sample. The data were calculated by averaging values after measuring at three different points on each sample.

\section{RESULTS}

\section{Analyses of Laccase-Catalyzed Dyes Toward Monomer Oxidation in Water Solutions at Acid and Alkaline pH}

The ability of POXA1b laccase to catalyze dye formation when incubated with seven commercially available compounds was first assayed in aqueous solution in both acidic and alkaline environments.

Dye generation catalyzed by laccase after different periods of incubation was observed, and the spectrum of each solution was monitored by visible spectrophotometry (Figure 1). Heatinactivated laccase was used as a negative control to monitor the spontaneous oxidation reaction, which occurred in the absence of laccase activity in the dyeing solution (Supplementary Figure S1). Color generation through spontaneous and laccase-induced reactions was measured by means of colorimetric analysis, and the related CIELAB coordinates $\mathrm{L}^{\star}, \mathrm{a}^{\star}$, and $\mathrm{b}^{*}$ were also reported (Figure 2 ).

For resorcinol (Figure 1A), the color of solution changed from colorless to orange during enzymatic oxidation at $\mathrm{pH}$ 9; however, no remarkable absorption band in the visible region was detected under acidic conditions. Conversely, an evident increase in absorption in the visible spectrum with a peak centered at around $490 \mathrm{~nm}$ was observed during the enzymatic incubation under alkaline conditions up to $5 \mathrm{~h}$. After $24 \mathrm{~h}$, the total absorption still increased, but this peak was not more detectable, and two others appeared centered at about 420 and $580 \mathrm{~nm}$.

The visible spectra of $p$-phenylenediamine oxidation exhibited a remarkable increase in absorption intensity centered in the 


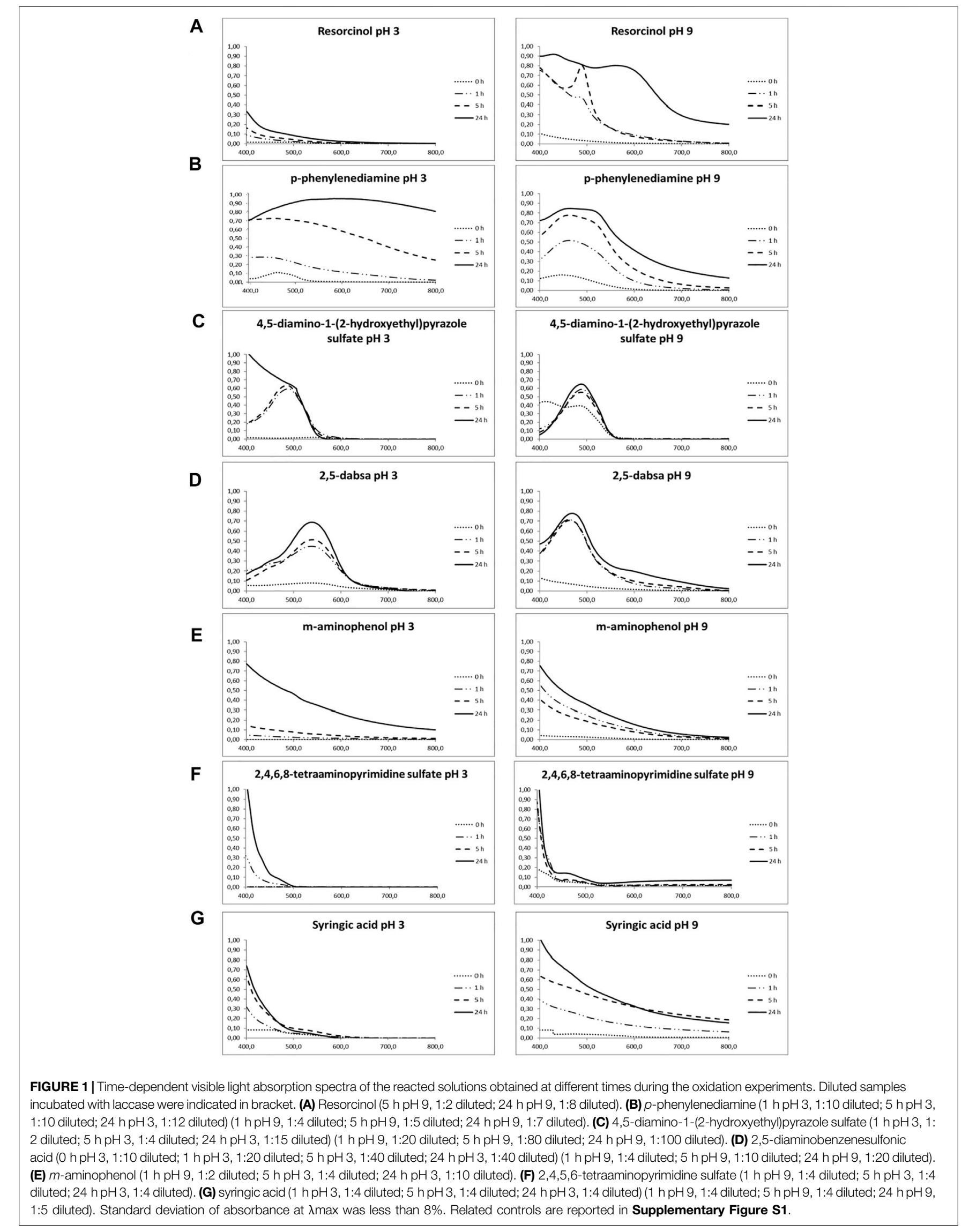




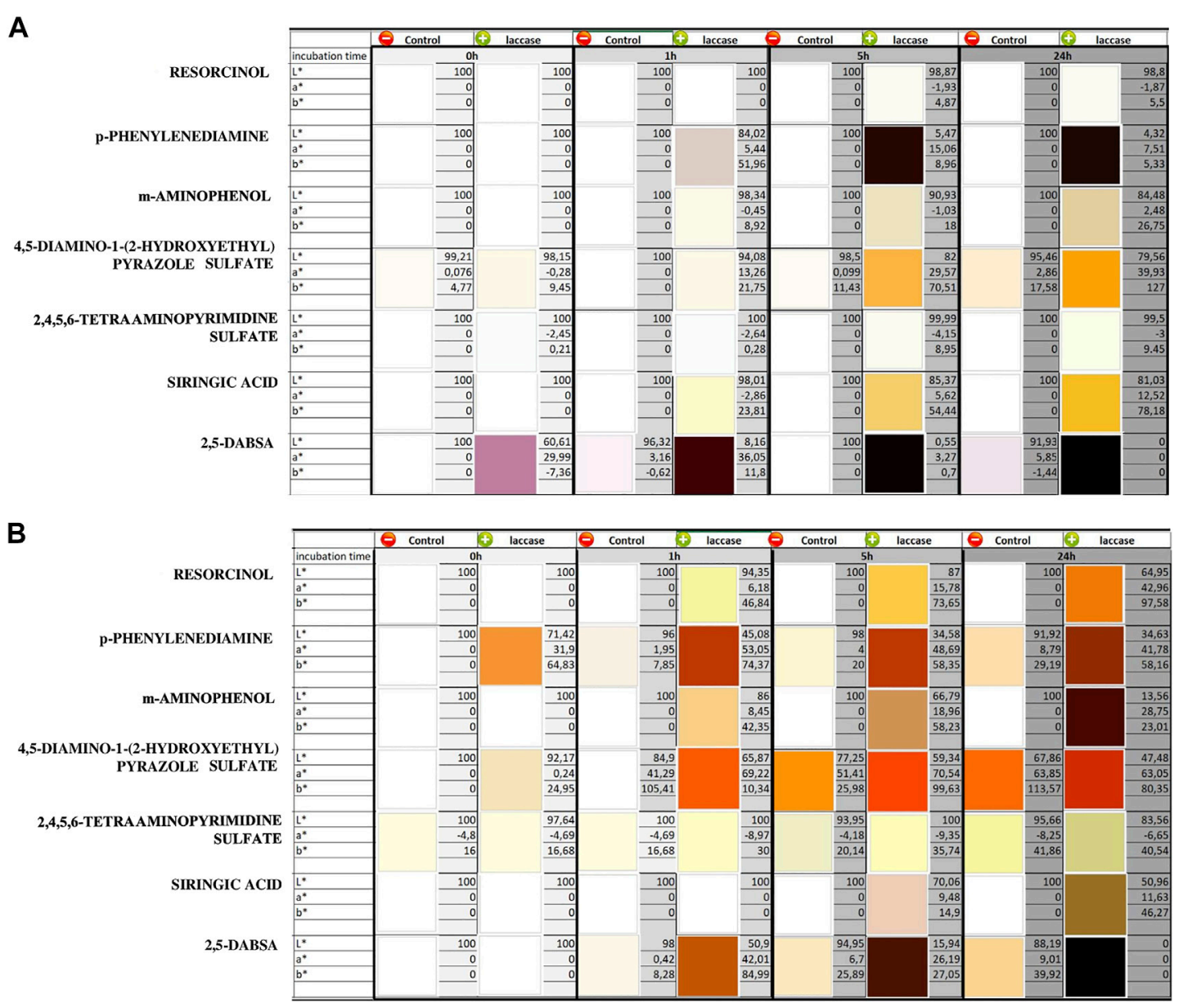

FIGURE 2 | CIELAB color values and related color images of the reacted solutions containing one monomer at different times at $\mathrm{pH} 3$ (A) and pH 9 (B). The solutions were evaluated at different time of laccase incubation $(0,1,5$, and $24 \mathrm{~h})$. The controls were analyzed under the same conditions by means of heat-inactivated laccase. The standard deviations were less than 3 units of $\Delta \mathrm{E}$ for each color value measurement.

range from 400 to $470 \mathrm{~nm}$, in both $\mathrm{pH}$ conditions, due to the electronic transition of quinoneimine structures in the polymer chains (Figure 1B). A red shift occurred after $24 \mathrm{~h}$ of incubation under acidic conditions, whereas a second peak at 500-550 nm appeared and increased during the incubation time under alkaline conditions. The color solution changed to dark brown under acidic conditions and red/light brown under alkaline conditions.

Spectrophotometric analysis of 4,5-diamino-1-(2hydroxyethyl)pyrazole sulfate displayed a peak at about $490 \mathrm{~nm}$, starting from the first hour of laccase action (Figure 1C). As the reaction continued, the absorbance remarkably increased, keeping the same curve shape for all the time points but $24 \mathrm{~h}$ at $\mathrm{pH} \mathrm{3}$, when the absorbance tended to increase in the ultraviolet wavelength direction. The color variation of the solution in the acid environment was not marked well, and a dark brown hue was detectable at $24 \mathrm{~h}$ of enzyme incubation at $\mathrm{pH} 9$.

POXA1b was also able to oxidize 2,5diaminobenzenesulfonic acid under both acidic and alkaline conditions (Figure 1D). In fact, a remarkable increase in absorption intensity was measured, with peaks centered at around 540 and $460 \mathrm{~nm}$ at $\mathrm{pH} 3$ and $\mathrm{pH} \mathrm{9,}$ respectively. Treatments at both $\mathrm{pH}$ produced colored products from cyclamen to dark violet/brown under the acidic condition and from orange to dark brown in the alkaline solution.

The laccase oxidation of $m$-aminophenol, 2,4,5,6tetraaminopyrimidine sulfate, and syringic acid showed similar patterns: a rapid decrease in absorbance with the wavelength increasing in the visible spectrum (Figure 1 from E to G). From the absorption spectra presented in Figure 1E, we can clearly observe that the reactions, under both $\mathrm{pH}$ conditions, increased the light absorption ability and that the enhancement of light absorption increased with the increase in the reaction time. The light absorption was higher close to the ultraviolet range and decreased with increasing wavelength.

As far as $m$-aminophenol is concerned, a faint color variation was detectable at $\mathrm{pH} 3$ only after $24 \mathrm{~h}$ of enzyme incubation, whereas brown color was produced after $5 \mathrm{~h}$ in the alkaline 


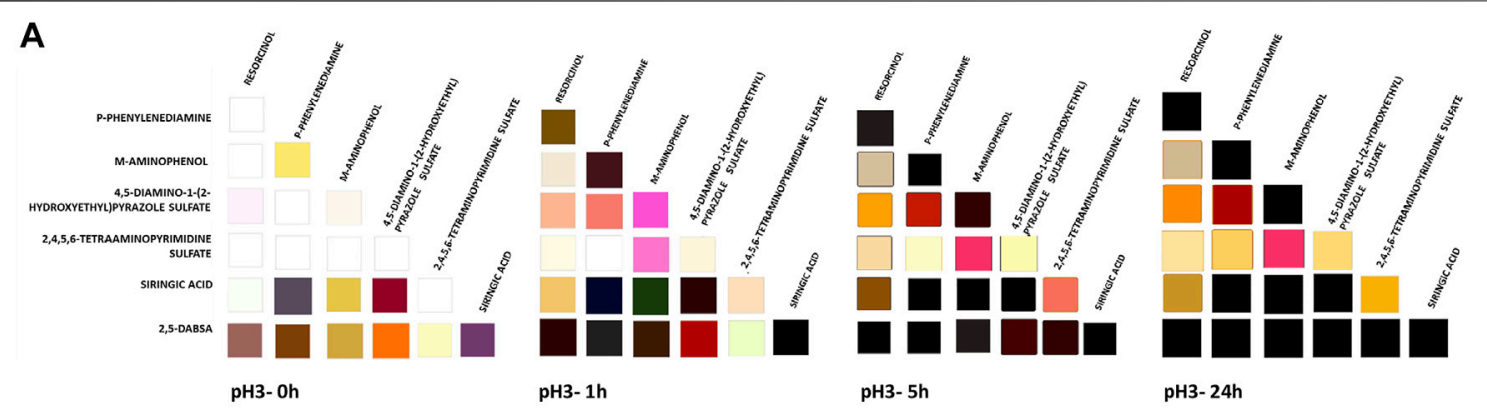

B

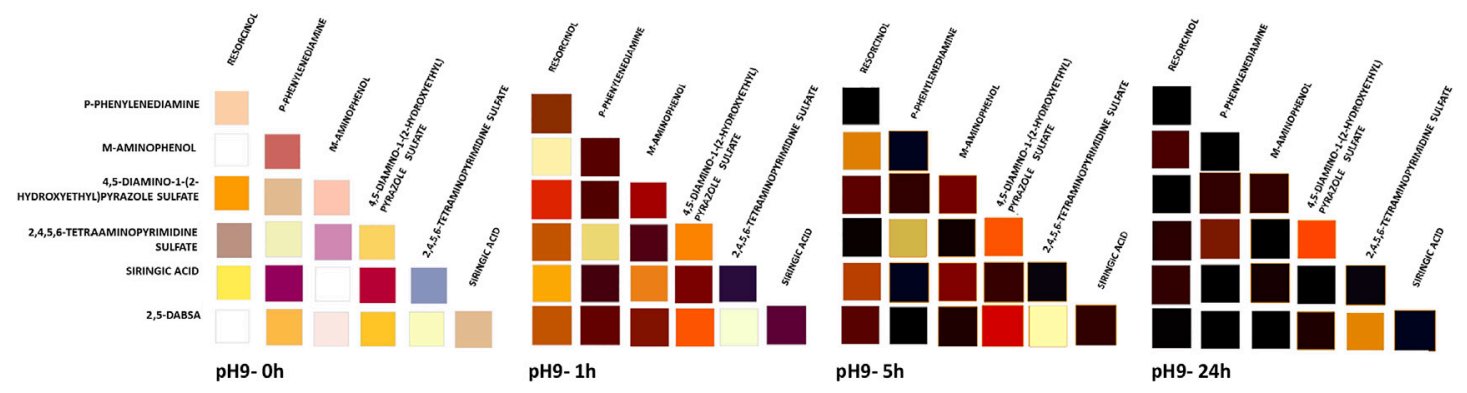

FIGURE 3 | Colors obtained in the enzymatic dyeing at $\mathrm{pH} 3 \mathbf{( A )}$ and pH 9 (B) of heteromolecular mixture. The solutions were evaluated at different time of laccase incubation $(0,1,5$, and 24 h). The corresponding CIELAB values are reported in Supplementary Table S2.

environment. A more pronounced yellow color was originated from the enzymatic solution containing syringic acid at $\mathrm{pH} 3$ after $5 \mathrm{~h}$, whereas dark beige was produced at $\mathrm{pH} 9$.

Finally, laccase seems to be inactive on 2,4,5,6tetraaminopyrimidine sulfate at both $\mathrm{pH}$ because no detectable difference occurred with respect to the controls. Considering the whole of reactions, it is not possible to rule out the existence of spontaneous oxidation in the absence of the enzyme, but for most of the compounds, a significant increase in the reaction rate is observable in the presence of laccase for both $\mathrm{pH}$ conditions. Most of the precursors gave colorless or slightly colored solutions when dissolved in water in the absence of oxidizers (Figure 2). On the other hand, addition of laccase resulted in visible color generation. Overall, the laccase-induced oxidation rate was higher in alkaline conditions than in the acidic ones, and the generated colors related to each compound at both $\mathrm{pH}$ significantly differed from each other in CIELAB coordinates.

\section{Increasing the Color Palette Through the Oxidation of Heteromolecular Mixture}

The synthesis of new colors was achieved by mixing of two monomers in aqueous solution in a 1:1 $\mathrm{M}$ ratio. Analogously to the previous experiments, all the twenty-one combinations derived from the coupling of the seven precursors were incubated with laccase at both $\mathrm{pH} 3$ and $\mathrm{pH} 9$.

Since the color related to the monomer polymerization changes with polymerization extent, which, in turn, depends on the polymerization time, the CIELAB and visible spectra of each reaction were measured over time. The color contribution, due to the autoxidation tendency of the heteromolecular mixtures, was evaluated by incubating each solution with heatinactivated laccase (Supplementary Table S2). The color parameters attained by the oxidation of the heteromolecular mixtures were also compared with the values related to the same blending solutions, whose monomers had been previously oxidized separately (Supplementary Table S3).

In the presence of the enzyme, a significant increase in the reaction rate was observed after $1 \mathrm{~h}$ of incubation of all heteromolecular mixtures under both $\mathrm{pH}$ conditions (Figure 3). In most of the cases, a significant color variation was also detected immediately after the start of the reaction (time 0).

After $5 \mathrm{~h}$ of laccase incubation, all solutions, derived by using the combinatorial approach, became colored. As the reaction continued, many of the colors darkened over time due to the extent of the polymerization degree and the increase in polymer concentration in the solution.

Taking all the results together, the color palette was expanded by using the combinatorial approach with respect to the color achieved by oxidation of the single monomers. All these results were used for wood fiber dyeing with the aim of evaluating the performances of each solution. A different effect is conceivable by applying each one.

\section{In situ Dyeing of Wood Fibers}

Once defined all the combination for color synthesis, the use of laccase to catalyze the in situ dyeing of wood fibers was 


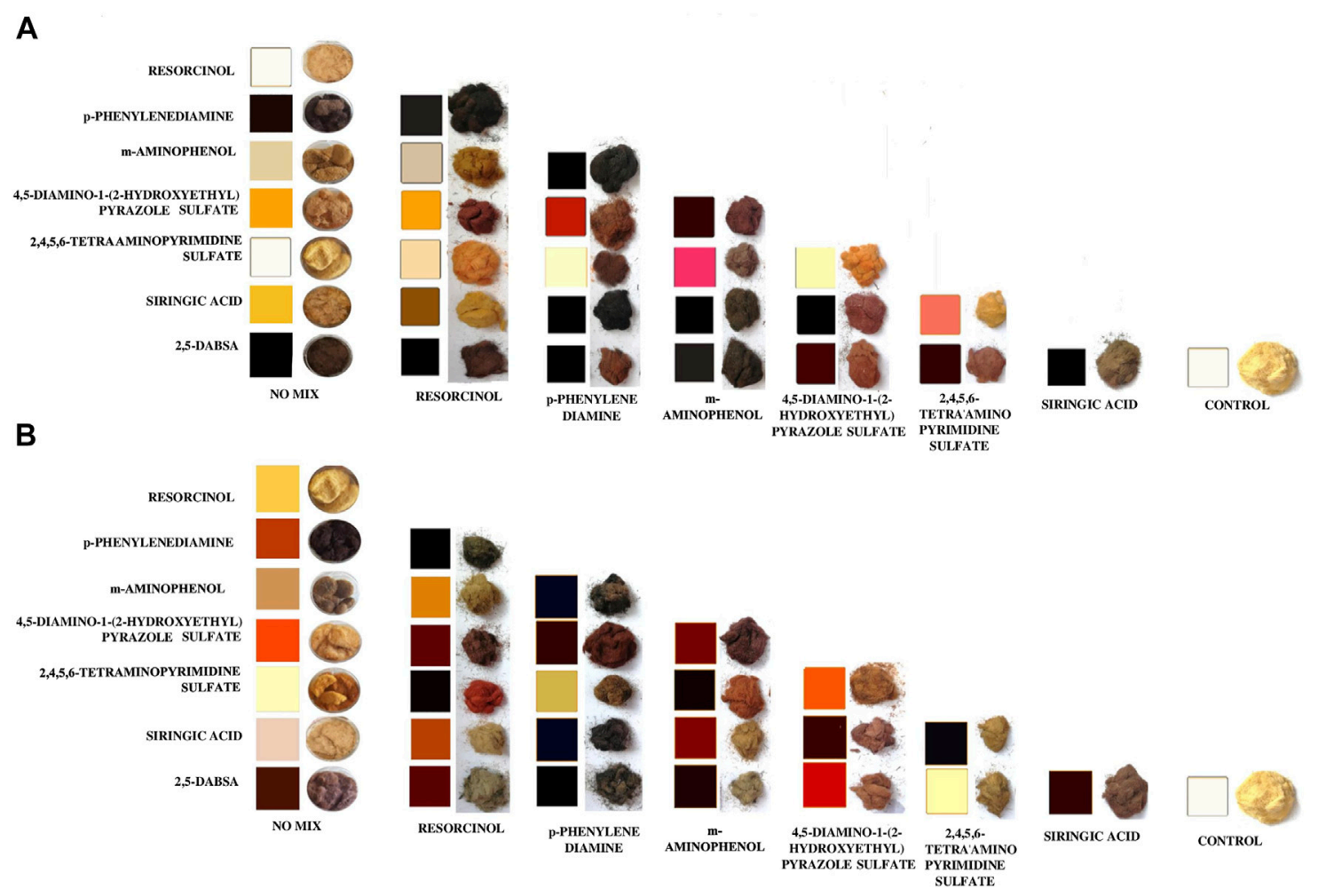

FIGURE 4 | Wood fibers dyed through laccases incubation together with single or coupled monomers in a dyeing bath at pH 3 (A) and pH 9 (B). The wood fiber color of each combination was compared with the color related to the corresponding solution.

investigated for each monomer and for each heteromolecular mixture. To this aim, the reactions were performed in a volume of $50 \mathrm{ml}$ per $1.5 \mathrm{~g}$ of dried fiber, sufficient to cover the wood fiber bulk. The enzyme incubation was kept for $24 \mathrm{~h}$, in order to achieve the highest yield of colored products on the fiber. The tests were performed under mild reaction conditions: $22 \pm 3^{\circ} \mathrm{C}$, $1.33 \pm 0.01 \mathrm{~atm}$, without stirring aqueous solution, and without any mordant or penetration agent.

Wood fibers treated with laccases only in the absence of oxidizable precursors led to no visible color change. Treatments comprising precursors in the absence of enzyme resulted in no color change across the fibers. In most of the cases, incubation of wood-based bulk with laccases and precursors resulted in evident in situ dyeing of the fibers.

A diverse range of colors and depths of shade were achieved and homogeneously distributed on the inner and outer parts of the bulk fiber, whereas only few of the coloring solutions were not able to stain the wood fibers. According to the results reported in Figure 4, the reaction products showed remarkable dyeing performances, in terms of intensity of color on the fibers. A variety of orange, red, brown, gray, and black colors was produced. Moreover, one dark green and one dark violet were also synthetized in situ. It has also been noticed that some of the dyes generated on the wood fibers totally differed from the color solution related to the corresponding reaction in liquid.

In most of the treated samples, the surface of each wood fiber was homogeneously dyed without uncolored spots. This finding is relevant in comparison with the dyed MDFs that are currently present on the market, whose surface has several uncolored spots on the MDF surface.

\section{Color Fastness of Water Analysis}

Dyed wood fiber samples were subjected to a washing cycle to evaluate the color resistance on this porous material. Undyed or meagerly colored samples were not tested for color fastness. From the data obtained (Table 1), it is possible to detect a slight color reduction of all dyed samples after soaking in hot water. $\Delta \mathrm{E}$ values were calculated for the colored samples before and after the treatment. $\Delta \mathrm{E}$ values of all treated samples were less than 7 . In particular, the fastness experiment had a relatively noticeable impact on the $\mathrm{L}^{\star}$ value, along with a minor impact on $\mathrm{a}^{*}$ and $\mathrm{b}^{*}$ (Supplementary Table S1) (Deng et al., 2009; Zhu et al., 2018).

\section{Water Contact Angle on the Wood Fibers}

The water contact angle was measured with a deionized water droplet on the surface of the form-pressed dried sample at $22 \pm$ $3^{\circ} \mathrm{C}$ (Table 2). As predictable, water in contact with the dried surface of the form-pressed wood fibers was rapidly absorbed (providing that the contact angle tends to be zero). The $20 \mu \mathrm{L}$ water was absorbed in $<10 \mathrm{~s}$ by a consistent number of wood fiber surfaces. However, for eighteen of the samples, the contact angles range from $57^{\circ}$ to $92^{\circ}$. The larger the wetting tendency, the smaller will be the contact angle or the surface tension. The time for drop absorption of six samples was less than $1 \mathrm{~min}$, whereas the water 
TABLE 1 | Color fastness properties of enzymatically dyed wood fibers. The $\Delta \mathrm{E}$ values were determined by measuring the CIELAB before and after washing treatment (reported in Supplementary Table S1).

Combined precursors
resorcinol; $p$-phenylenediamine
resorcinol; $m$-aminophenol
resorcinol; 2,4,5,6-tetraaminopyrimidine sulfate
resorcinol; 4,5-diamino-1-(2-hydroxyethyl)pyrazole sulfate
resorcinol; syringic acid
resorcinol; 2,5-diaminobenzenesulfonic acid
p-phenylenediamine; $m$-aminophenol
p-phenylenediamine; 2,4,5,6-tetraaminopyrimidine sulfate
p-phenylenediamine; syringic acid
p-phenylenediamine; 2,5-diaminobenzenesulfonic acid
p-phenylenediamine; 4,5-diamino-1-(2-hydroxyethyl)pyrazole sulfate
$m$-aminophenol; syringic acid
$m$-aminophenol; 2,5-diaminobenzenesulfonic acid
4,5-diamino-1-(2-hydroxyethyl)pyrazole sulfate; $2,4,5,6$-tetraaminopyrimidine sulfate
4,5-diamino-1-(2-hydroxyethyl)pyrazole sulfate; 2,5 -diaminobenzenesulfonic acid
4,5-diamino-1-(2-hydroxyethyl)pyrazole sulfate; syringic acid
2,4,5,6-tetraaminopyrimidine sulfate; syringic acid
2,4,5,6-tetraaminopyrimidine sulfate; 2,5 -diaminobenzenesulfonic acid
$m$-aminophenol; 4,5-diamino-1-(2-hydroxyethyl)pyrazole sulfate
$m$-aminophenol; $2,4,5,6$-tetraaminopyrimidine sulfate
syringic acid; 2,5 -diaminobenzenesulfonic acid

$\Delta E$ colors achieved at pH 3

pH 3

7
1
4
7
-
6
3
4
1
6
5
6
7
5
6
6
-
5
4
4
6

$\Delta \mathrm{E}$ colors achieved at pH 9

The standard deviation was less than 1 unit of $\Delta E$, for each measurement.

drops in contact with the surface of seven of them were still detectable after $5 \mathrm{~min}$ (Figure 5). Some of the wood fiber surfaces absorbed water in more than $15 \mathrm{~min}$, in particular those treated with solutions containing $m$-aminophenol, 2,4,5,6tetraaminopyrimidine sulfate, or 4,5-diamino-1-(2hydroxyethyl)pyrazole sulfate. In detail, the incubation of wood fibers with laccase and 4,5-diamino-1-(2-hydroxyethyl) pyrazole sulfate at $\mathrm{pH} 9$ allowed the surfaces not to absorb water drops for about $70 \mathrm{~min}$.

\section{DISCUSSION}

Laccases can efficiently catalyze polymerization of phenolic and aromatic moieties. Polymerization is initiated by the formation of a radical cation, followed by intermolecular attack to produce dimers (Mikolasch and Schauer, 2009). Colored oligomers and polymers can be synthesized from dimers, miming the natural phenomenon responsible for synthesis of flavonoid polymers. Finally, monomers can be blended into oxidative solutions, thus promoting synthesis of heteropolymers and expanding the color varieties (Jeon et al., 2010; Sousa et al., 2016).

In this work, an innovative and environmentally-friendly process for wood fiber dyeing, based on laccase-catalyzed oxidation, was developed. Seven monomers were selected, from a list of commercially available precursors, for their chemical structures and for their oxidation potential.

Resorcinol is a non-expensive catechol isomer that has already been used as a substrate to produce colored homopolymers and heteropolymers via laccase-catalyzed oxidation and has also been exploited in laccase-catalyzed fiber dyeing (Durairaj, 2005; Sun et al., 2013; Reena et al., 2014; Sýs et al., 2017).
p-Phenylenediamine is a monocyclic aryl amine compound, a white to light purple powder, which, when oxidized, turns red first, then brown, and then finally black on exposure to air. It has been used in the past as an ingredient of oxidative hair coloring products or as a textile dye and also as a photographic developing agent (Latha Saranya et al., 2014).

4,5-diamino-1-(2-hydroxyethyl)pyrazole sulfate is a promising candidate as new primary intermediates, considering that it may decrease allergenic risks (Ramadan et al., 2021). As a fact, several patents on diaminopyrazoles and triaminopyrazoles as primary intermediates has been recently granted (Cotteret, 2002; Chassot and Braun, 2003; Ernst et al., 2006; Vidal and Fadli, 2007; Lim et al., 2008; Thuring et al., 2014).

2,5-diaminobenzenesulfonic acid has been already used successfully for the in situ coloration of textile fibers, coupled to catechol and resorcinol (Calafell et al., 2007; Pezzella et al., 2016; Zerva et al., 2019). m-Aminophenol is already used to increase the number of polymer products because its polymerization mechanism is similar to that of aniline, thus differing from the widely used o-isomer (Dinç et al., 2014).

2,4,5,6-tetraaminopyrimidine, like other tetraaminopyrimidines and triaminopyrimidines, is a heterocyclic dye precursor considered as alternatives to carbocycles in dyes (Morel and Christie, 2011). Renewed interest in pyridine derivatives is indicated by claims as primary intermediates and as couplers in several patents.

Syringic acid is one of the abundant phenolic compounds present in several plants (Srinivasulu et al., 2018), which contributes to the structural integrity of the lignin. Its oxidation in the presence of laccase has been already reported (Jeon et al., 2012). The formation of oligo(phenylene oxide)s to 
TABLE 2 | Water contact angles (in grade) and drop adsorption time (in minutes) of dyed wood fibers.

\begin{tabular}{|c|c|c|c|c|c|c|c|c|}
\hline pH 3 & resorcinol & p-phenylenediamine & $m$-aminophenol & $\begin{array}{c}\text { 4,5-diamino-1-(2- } \\
\text { hydroxyethyl)pyrazole } \\
\text { sulfate }\end{array}$ & $\begin{array}{c}2,4,5,6- \\
\text { tetraaminopyrimidine } \\
\text { sulfate }\end{array}$ & $\begin{array}{l}\text { syringic } \\
\text { acid }\end{array}$ & $\begin{array}{c}2,5- \\
\text { diaminobenzenesulfonic } \\
\text { acid }\end{array}$ & Control \\
\hline resorcinol & $92^{\circ}(0.1 \mathrm{~min})$ & ---------- & --------- & --------- & --------- & --------- & ---------- & -------- \\
\hline$p$-phenylenediamine & $82.5^{\circ}(1.1 \mathrm{~min})$ & Spreading $^{\mathrm{a}}$ & --------- & -------- & --------- & -------- & -------- & --------- \\
\hline$m$-aminophenol & $57^{\circ}(0.1 \mathrm{~min})$ & $83^{\circ}$ (3.5 min) & Spreading $^{\mathrm{a}}$ & --------- & -.------ & --.----- & -------- & -.---.-- \\
\hline $\begin{array}{l}\text { 4,5-diamino-1-(2-hydroxyethyl) } \\
\text { pyrazole sulfate }\end{array}$ & $89^{\circ}(2.3 \mathrm{~min})$ & Spreading $^{a}$ & $80^{\circ}(17.0 \mathrm{~min})$ & $86^{\circ}(6.0 \mathrm{~min})$ & --------- & -------- & ---------- & --------- \\
\hline $\begin{array}{l}\text { 2,4,5,6-tetraaminopyrimidine } \\
\text { sulfate }\end{array}$ & Spreading $^{\text {a }}$ & $73^{\circ}(0.2 \mathrm{~min})$ & $88^{\circ}(19.0 \mathrm{~min})$ & $85^{\circ}(1.0 \mathrm{~min})$ & $86^{\circ}(5.30 \mathrm{~min})$ & ------- & --- & ------- \\
\hline syringic acid & Spreading $^{a}$ & $88^{\circ}$ (0.2 min) & $106^{\circ}(1.1 \mathrm{~min})$ & Spreading $^{\mathrm{a}}$ & Spreading $^{a}$ & Spreading ${ }^{a}$ & & -------- \\
\hline 2,5-diaminobenzenesulfonic acid & Spreading $^{a}$ & $72^{\circ}(0.2 \mathrm{~min})$ & Spreading $^{a}$ & Spreading $^{a}$ & Spreading $^{a}$ & Spreading $^{a}$ & Spreading $^{a}$ & Spreadinga \\
\hline pH 9 & resorcinol & $p$-phenylenediamine & $m$-aminophenol & $\begin{array}{l}\text { 4,5-diamino-1-(2- } \\
\text { hydroxyethyl)pyrazole } \\
\text { sulfate }\end{array}$ & $\begin{array}{c}2,4,5,6- \\
\text { tetraaminopyrimidine } \\
\text { sulfate }\end{array}$ & $\begin{array}{l}\text { syringic } \\
\text { acid }\end{array}$ & $\begin{array}{c}2,5- \\
\text { diaminobenzenesulfonic } \\
\text { acid }\end{array}$ & Control \\
\hline resorcinol & Spreading $^{\mathrm{a}}$ & ---------- & --------- & 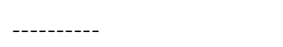 & -------- & --------- & -------- & ---------- \\
\hline$p$-phenylenediamine & Spreading ${ }^{a}$ & Spreading $^{a}$ & -------- & -.------ & --------- & --------- & -------- & --.------ \\
\hline$m$-aminophenol & Spreading ${ }^{a}$ & Spreading $^{a}$ & Spreading $^{\mathrm{a}}$ & -------- & --------- & --------- & --------- & --------- \\
\hline $\begin{array}{l}\text { 4,5-diamino-1-(2-hydroxyethyl) } \\
\text { pyrazole sulfate }\end{array}$ & Spreading $^{\mathrm{a}}$ & Spreading $^{\mathrm{a}}$ & $82^{\circ}$ (0.2 min) & $90^{\circ}$ (70.0 min) & -------- & & ---- & \\
\hline $\begin{array}{l}\text { 2,4,5,6-tetraaminopyrimidine } \\
\text { sulfate }\end{array}$ & $95^{\circ}(15.3 \mathrm{~min})$ & Spreading $^{a}$ & $77^{\circ}$ (0.2 min) & Spreading $^{\text {a }}$ & Spreading $^{\mathrm{a}}$ & ------- & -------- & -------- \\
\hline syringic acid & Spreading ${ }^{a}$ & Spreading ${ }^{a}$ & Spreading ${ }^{a}$ & Spreading ${ }^{a}$ & Spreading ${ }^{a}$ & Spreading ${ }^{a}$ & ---------- & --------- \\
\hline 2,5-diaminobenzenesulfonic acid & Spreading ${ }^{a}$ & Spreading ${ }^{a}$ & Spreading $^{a}$ & Spreading $^{a}$ & Spreading $^{a}$ & Spreading $^{a}$ & $85^{\circ}(24.0 \mathrm{~min})$ & Spreadinga \\
\hline
\end{tabular}

aSpreading: the water droplet is completely spread out/absorbed on the solid surface.

The standard deviation for each sample was less than $4 \%$. 


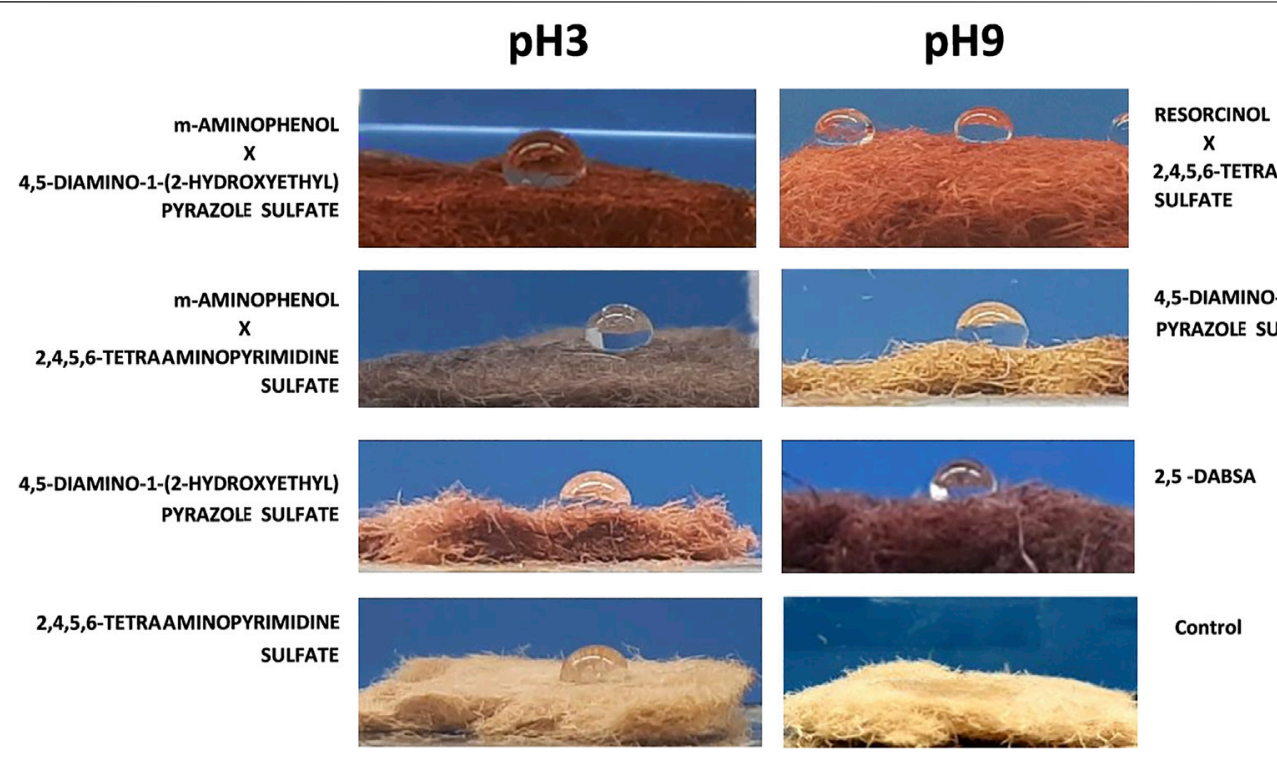

FIGURE 5 |Water drop depositions of the less absorbent wood fibers dyed with laccase and single precursor solutions or heteromolecular mixtures. 20 ul of water solution was dropped on the pressed fiber surface, and the picture was taken after $2 \mathrm{~s}$ from the deposition only for the droplet that was not immediately absorbed. The controls were incubated with the same enzymatic solutions at both $\mathrm{pH}$ in absence of precursors. They displayed the same behavior and one of them is reported in picture as reference.

hexamers has been observed, but the detailed characterization of the oligomers has not been well understood. The presence of syringic acid in lignin may act as a good substrate for fungal laccases, a fact that has great importance in the bioremediation and pulp industry (Abe et al., 2005). Furthermore, syringic acid-based synthetic lignin polymers have been prepared using laccase-catalyzed polymerization (Ikeda et al., 1996).

To get insights into heteromolecular mixture contribution to the colored products generated through laccase catalysis, products belonging to homopolymerization and heteropolymerization reactions under acidic and alkaline $\mathrm{pH}$ were compared. As it also happens in the case of the hair dyeing process, the reactions are mostly promoted by high $\mathrm{pH}$ (Da França et al., 2015), despite POXA1b catalytic properties, whose activity is increased under acidic conditions. The apparent incongruency can be explained by the structure of the active site of laccases and the different nature of non-phenolic substrates such as ABTS, which is generally used as a reference to measure laccase activity, with respect to the dye precursors. The active site of laccases contains four copper ions: a mononuclear copper ion (T1) and a trinuclear copper T2/T3 cluster (Janusz et al., 2020), and the catalysis follows three major steps: T1 copper is reduced by accepting electrons from the reducing substrate, then electrons are transferred from the mononuclear copper to the T2/T3 cluster, and finally molecular oxygen is reduced to water at the T2/T3 cluster.

As far as ABTS is concerned, whose oxidation does not involve protons and has a minimal redox potential, the activity of laccase decreases when $\mathrm{pH}$ increases, as a result of the possible involvement of inhibition at the T2/T3 center ( $\mathrm{Xu}, 1997)$. For a reducing substrate, whose oxidation involves protons, such as phenols, and has a significant redox potential dependence on $\mathrm{pH}$, the $\mathrm{pH}$ activity profile of laccase could increase, reflecting the $\mathrm{pH}$ induced redox potential change on both the T1 center and substrate (Xu, 1997).

Solubilization of two different monomers into the same solution results in the increasing rate of color formation. This effect is even more pronounced at $\mathrm{pH} 3$, where the reactivity of the monomers resulted remarkably reduced. A heteromolecular mixture also has the advantage of expanding the chromatic scale of the oxidized solutions. The production of new colors is not due to the presence of more than one homopolymer in the same solution, whose chromatic contribution would overlap, but are related to the formation of new heteropolymeric compounds. In fact, when the single monomer solutions are separately oxidized through laccases and then blended together, the samples display color values that differ from those obtained by oxidizing the monomers together in the same solution. Laccases can promote heteromolecular coupling reactions when two or more substrates are incubated together (Pilz et al., 2003; Jeon et al., 2012). Due to the nature of the products, the heteropolymers have a different absorption profile in comparison with the one with the presence of two homopolymers in the same solution.

As previously reported for other fibrous materials (Jeon et al., 2010; Pezzella et al., 2016; Kim et al., 2017; Agrawal et al., 2018; Prajapati et al., 2018), radical-induced polymerizations can take place in the fibers of porous materials and stably dye them through both the steric entrapment of the polymer and the chemical bonding occurring between the colored polymers and the fibers. Despite the absence of stirring and the mild reaction conditions, which allow containing the process costs, the color achieved in most of the reactions is homogeneously distributed over the entire surface of the fibers, even on the innermost part of the batch, keeping its "wooden natural" surface (Figure 6A). 


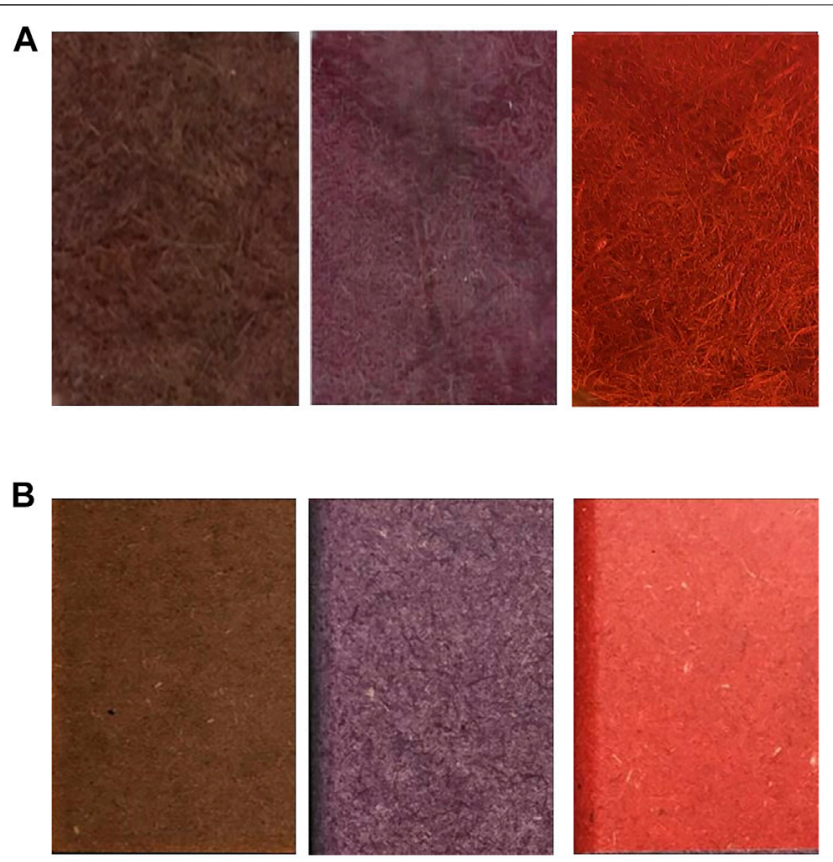

FIGURE 6 | Comparison of dried pressed wood fiber panels that were previously dyed toward laccase catalysis (A) with the commercially dyed MDF already present on the market (B)

Conversely, the stained fibers of commercially available MDFs are impregnated with organic coloring agents and are chemically bound to one another by a special resin and come in different shades (Figure 6B). As reported in the related datasheet, this variation may be seen on the same surface, between the two faces of the same panel, or between the different production batches or different thicknesses. To minimize this effect, the supply must be from a single production. The MDFs fabricated by using wood fibers dyed through laccase could provide a more homogeneous color surface and more reproducible batches with respect to the panels already present on the market. Moreover, the laccasebased dyeing process was found to be more sustainable than the organic-based one, which uses more recalcitrant and less effective dyestuffs.

The performances of the dyeing process, together with its mild conditions, the presence of natural catalyzer, and the absence of recalcitrant chemicals, make the wood fiber dyeing through laccase highly advantageous and ecologically more sustainable. It has been already quoted that some of the dyes generated on the wood fibers display a color significantly different from their dye bath. Since lignin can be a substrate of laccases (Perna et al., 2020), lignin itself, or monomers obtained from its oxidative degradation and can be included in the polymerization reaction; thus, some dyes synthetized in situ differ completely from their liquid counterpart. However, the reported difference in color values points out the need to test the conditions of each reaction on wood fibers in order to assess the achievement of a specific color point.

The color generated from the laccase oxidative reaction generally lead to achieving a satisfactory fastness on different materials such as cotton, wool, silk, leather, and nylon (Kim et al., 2007; Sun et al., 2014; Pezzella et al., 2016; Prajapati.et al., 2018; Antunes Barros et al., 2019; Atav et al., 2021). The wood fiber's color resistance to fading and running confirms a strong binding between the dye and the fiber, with satisfactory color fastness of water property (Zhu et al., 2018) in comparison with previous studies on the fastness of the dyed wood material (Deng et al., 2009). From this point of view, a further advantage of the use of laccases in the dyeing process could be provided by their grafting action on the wood fibers, which could allow the covalent binding of the dye to the ligneous surface (Felby et al., 2002; Schubert et al., 2015; Slagman et al., 2018). Further studies to better explain this phenomenon are still needed.

Some of the laccase-treated wood fibers were less permeable to water (Francisca et al., 2003; Malkov et al., 2004; Iovino et al., 2018). This characteristic shed light to the possibility to prepare an innovative eco-friendly MDF with less impermeabilization agents based on the petrochemical industry.

The improved characteristics of laccase-treated wood fibers over conventional processing methods allow adopting a simpler and milder manufacturing process that limits the use of harsh chemicals and energy consumption and allow producing innovative MDFs three dimensionally dyed with natural textures, addressing the industrial and technical needs required by the market.

\section{DATA AVAILABILITY STATEMENT}

The original contributions presented in the study are included in the article/Supplementary Material; further inquiries can be directed to the corresponding author.

\section{AUTHOR CONTRIBUTIONS}

VL designed and directed the study. $\mathrm{AC}$ and $\mathrm{AD}$ performed the experiments. AC expressed and purified the enzymes. AD analyzed the spectra. AC and VL analyzed the data. VL wrote the manuscript with input from all authors.

\section{ACKNOWLEDGMENTS}

The authors acknowledge Prof. Giovanni Sannia of the Department of Chemical Science of the University of Naples Federico II for his critical reading of the article and Carmela di Lauro and Raffaele Lettera for their valuable suggestions and help.

\section{SUPPLEMENTARY MATERIAL}

The Supplementary Material for this article can be found online at: https://www.frontiersin.org/articles/10.3389/fbioe.2021.778971/ full\#supplementary-material 


\section{REFERENCES}

Abe, T., Masai, E., Miyauchi, K., Katayama, Y., and Fukuda, M. (2005). A Tetrahydrofolate-dependent $\mathrm{O}$-Demethylase, LigM, Is Crucial for Catabolism of Vanillate and Syringate in Sphingomonas Paucimobilis SYK6. J. Bacteriol. 187, 2030-2037. doi:10.1128/JB.187.6.2030-2037.2005

Agrawal, K., Chaturvedi, V., and Verma, P. (2018). Fungal Laccase Discovered but yet Undiscovered. Bioresour. Bioproc. 5, 1-12. doi:10.1186/s40643-018-0190-z

Agustin, M. B., Carvalho, D. M., Lahtinen, M. H., Hilden, K., Lundell, T., and Mikkonen, K. S. (2021). Laccase as a Tool in Building Advanced Lignin-Based Materials. ChemSusChem 14, 4615-4635. doi:10.1002/cssc.202101169

Andlar, M., Rezić, T., Marđetko, N., Kracher, D., Ludwig, R., and Šantek, B. (2018). Lignocellulose Degradation: an Overview of Fungi and Fungal Enzymes Involved in Lignocellulose Degradation. Eng. Life Sci. 18, 768-778. doi:10.1002/elsc.201800039

Antunes Barros, A. A., da Rocha Cardoso, A. P., Rodrigues, A. C., dos Santos Marinho da Silva, C. J., and Zille, A. (2019). Optimizing Enzymatic Dyeing of Wool and Leather. SN Appl. Sci. 1, 1232. doi:10.1007/s42452-019-1241-6

Atav, R., Buğdaycı, B., Bozkurt, Ö., Yıldız, A., Güneş, E., and Yakın, İ. (2021). Laccase-catalyzed Enzymatic Dyeing of Cotton Fabrics. Textile Res. J., 004051752110069. doi:10.1177/00405175211006937

Berglund, L., and Rowell, R. M. (2005). Wood Composites. Boca Raton: CRC Press.

Calafell, M., Díaz, C., Hadzhiyska, H., Gibert, J. M., Dagà, J. M., and Tzanov, T. (2007). Bio-catalyzed Coloration of Cellulose Fibers. Biocatal. Biotransformation 25, 336-340. doi:10.1080/10242420701379874

Chassot, L., and Braun, H. J. (2003). Diaminopyrazole Derivatives and Dyes Containing Said Compounds. U.S. Patent No. 6,600,050 B2 (Darmstadt, DE: United States Patent).

Cotteret, J. (2002). Composition for Oxidation Dyeing of Keratinous Fibres Comprising an Amino Pyrazole and a Cellulose Compound. W.O. Patent No. 02/051372 A2 (Paris, FR: Patent Cooperation Treaty).

da França, S., Dario, M., Esteves, V., Baby, A., and Velasco, M. (2015). Types of Hair Dye and Their Mechanisms of Action. Cosmetics 2, 110-126. doi:10.3390/ cosmetics 2020110

Deng, S. P., Ye, C. X., Chen, X. Y., and Bian, L. P. (2009). Study on the Effect of Color Fastness of Water about Three Dyeing Auxiliary to Dyed Veneer and its Mechanism Analysis by FTIR Methods. J. Fujian Coll. For. 29, 45-48. doi:10.13324/j.cnk.jfcf.2009.01.003

Dinç, C. Ö., Yalçınkaya, S., Altuntaş, H., and Çolak, N. (2014). Synthesis and Characterization of Poly(m-Aminophenol)-Succinat. Designed Monomers Polym. 17, 629-636. doi:10.1080/15685551.2014.907617

Durairaj, R. B. (2005). Resorcinol: Chemistry, Technology and Applications. New York, United States: Springer.

Ernst, G., Frietze, W., and Simpson, T. (2006). Novel Pyrazole Derivatives and Their Use as Modulators of Nicotinic Acetylcholine Receptors. W.O. Patent No 068,591 (Södertälje, SE: Patent Cooperation Treaty).

Euring, M., Rühl, M., Ritter, N., Kües, U., and Kharazipour, A. (2011). Laccase Mediator Systems for Eco-Friendly Production of Medium-Density Fiberboard (MDF) on a Pilot Scale: Physicochemical Analysis of the Reaction Mechanism. Biotechnol. J. 6, 1253-1261. doi:10.1002/biot.201100119

Felby, C., Hassingboe, J., and Lund, M. (2002). Pilot-scale Production of Fiberboards Made by Laccase Oxidized wood Fibers: Board Properties and Evidence for Cross-Linking of Ligninfiberboards Made by Laccase Oxidized wood Fibers: Board Properties and Evidence for Cross-Linking of Lignin. Enzyme Microb. Tech. 31, 736-741. doi:10.1016/s0141-0229(02)00111-4

Francisca, F. M., Rinaldi, V. A., and Santamarina, J. C. (2003). Instability of Hydrocarbon Films over mineral Surfaces: Microscale Experimental Studies. J. Environ. Eng. 129, 12 1120-1128. doi:10.1061/(asce)0733-9372(2003)129:12(1120)

Hu, J., Du, W., Ji, X., Yuan, B., Liu, Y., and Guo, M. (2016). The Chemistry, Morphology, crystal Structure and Hydrophilicity Properties of wood Fibers Treated by a Magnetic Immobilized Laccase-Mediator System. RSC Adv. 6, 32572-32579. doi:10.1039/c6ra00740f

Ikeda, R., Uyama, H., and Kobayashi, S. (1996). Novel Synthetic Pathway to a Poly(phenylene Oxide). Laccase-Catalyzed Oxidative Polymerization of Syringic Acid. Macromolecules 29, 3053-3054. doi:10.1021/ma951810b

Iovino, M., Pekárová, P., Hallett, P. D., Pekár, J., Lichner, L., Mataix-Solera, J., et al. (2018). Extent and Persistence of Soil Water Repellency Induced by Pines in
Different Geographic Regions. J. Hydrol. Hydromech. 66, 360-368. doi:10.2478/ johh-2018-0024

Janusz, G., Pawlik, A., Świderska-Burek, U., Polak, J., Sulej, J., Jarosz-Wilkołazka, A., et al. (2020). Laccase Properties, Physiological Functions, and Evolution. Ijms 21, 966. doi:10.3390/ijms21030966

Jeon, J.-R., Baldrian, P., Murugesan, K., and Chang, Y.-S. (2012). Laccase-catalysed Oxidations of Naturally Occurring Phenols: from In Vivo Biosynthetic Pathways to green Synthetic Applications. Microb. Biotechnol. 5, 318-332. doi:10.1111/j.1751-7915.2011.00273.x

Jeon, J.-R., Kim, E.-J., Murugesan, K., Park, H.-K., Kim, Y.-M., Kwon, J.-H., et al. (2010). Laccase-catalysed Polymeric Dye Synthesis from Plant-Derived Phenols for Potential Application in Hair Dyeing: Enzymatic Colourations Driven by Homo- or Hetero-Polymer Synthesis. Microb. Biotechnol. 3, 324-335. doi:10.1111/j.1751-7915.2009.00153.x

Kim, S., Lee, H., Kim, J., Oliveira, F., Souto, P., Kim, H., et al. (2017). Laccasemediated Grafting of Polyphenols onto Cationized Cotton Fibers to Impart UV protection and Antioxidant Activities. J. Appl. Polym. Sci. 135, 45801. doi:10.1002/APP.45801

Kim, S., Moldes, D., and Cavaco-Paulo, A. (2007). Laccases for Enzymatic Colouration of Unbleached Cotton. Enzyme Microb. Tech. 40, 1788-1793. doi:10.1016/j.enzmictec.2007.01.002

Kumar, D., Kumar, A., Sondhi, S., Sharma, P., and Gupta, N. (2018). An Alkaline Bacterial Laccase for Polymerization of Natural Precursors for Hair Dye Synthesis. 3 Biotech. 8, 182. doi:10.1007/s13205-018-1181-7

Latha Saranya, C. H., Gurupadayya, B. M., Kinnera, K., and Thejaswini, J. C. (2014). Spectrophotometric Determination of p-phenylenediamine in Hair Dyes. Turk. J. Pharm. Sci. 11, 295-306.

Lettera, V., Piscitelli, A., Leo, G., Birolo, L., Pezzella, C., and Sannia, G. (2010). Identification of a New Member of Pleurotus Ostreatus Laccase Family from Mature Fruiting Body. Fungal Biol. 114, 724-730. doi:10.1016/ j.funbio.2010.06.004

Lim, M., Zhang, G., and Murphy, B. P. (2008). Novel Hair Colouring Compositions for Use in Oxidative Hair Dyeing. E.P. Patent No. 1,982,748 A2. Cincinnati, $\mathrm{OH}$ : European Patent Application.

López, A. M. Q., Silva, A. L. S., and Santos, E. C. L. (2017). The Fungal Ability for Biobleaching/biopulping/bioremediation of Lignin-like Compounds of AgroIndustrial Raw Material. Quim. Nova 40, 916-931. doi:10.21577/01004042.20170067

Malkov, S. Y., Kuzmin, V. A., Baltakhinov, V. P., and Tikka, P. (2004). Modelling the Process of Water Penetration into Softwood Chips. J. Pulp Pap. Sci. 25, 123-129.

Mikolasch, A., and Schauer, F. (2009). Fungal Laccases as Tools for the Synthesis of New Hybrid Molecules and Biomaterials. Appl. Microbiol. Biotechnol. 82, 605-624. doi:10.1007/s00253-009-1869-z

Morel, O. J. X., and Christie, R. M. (2011). Current Trends in the Chemistry of Permanent Hair Dyeing. Chem. Rev. 111, 2537-2561. doi:10.1021/cr1000145

Nasir, M., Gupta, A., Hossen Beg, M. D., Chua, G. K., and Asim, M. (2014). Laccase Application in Medium Density Fibreboard to Prepare a Bio-Composite. RSC Adv. 4, 11520-11527. doi:10.1039/C3RA40593A

Nasir, M., Hashim, R., Sulaiman, O., Gupta, A., Khan, T. A., Jawaid, M., et al. (2016). Natural Fiber Improvement by Laccase; Optimization, Characterization and Application in Medium Density Fiberboard. J. Nat. Fibers 14, 379-389. doi:10.1080/15440478.2016.1212759

Perna, V., Meyer, A. S., Holck, J., Eltis, L. D., Eijsink, V. G. H., and Wittrup Agger, J. (2020). Laccase-Catalyzed Oxidation of Lignin Induces Production of H2O2. ACS Sustain. Chem. Eng. 8, 831-841. doi:10.1021/acssuschemeng.9b04912

Pezzella, C., Giacobbe, S., Giacobelli, V. G., Guarino, L., Kylic, S., Sener, M., et al. (2016). Green Routes towards Industrial Textile Dyeing: A Laccase Based Approach. J. Mol. Catal. B: Enzymatic 134, 274-279. doi:10.1016/ j.molcatb.2016.11.016

Pilz, R., Hammer, E., Schauer, F., and Kragl, U. (2003). Laccase-catalysed Synthesis of Coupling Products of Phenolic Substrates in Different Reactors. Appl. Microbiol. Biotechnol. 60, 708-712. doi:10.1007/s00253-002-1181-7

Prajapati, C. D., Smith, E., Kane, F., and Shen, J. (2018). Laccase-catalysed Coloration of Wool and Nylon. Coloration Technol. 134, 423-439. doi: $10.1111 /$ cote. 12350

Ramadan, M., Aly, A. A., El-Haleem, L. E. A., Alshammari, M. B., and Bräse, S. (2021). Substituted Pyrazoles and Their Heteroannulated Analogs-Recent 
Syntheses and Biological Activities. Molecules 26, 4995. doi:10.3390/ molecules26164995

Reena, Dhall, P., Kumar, R., and Kumar, A. (2014). Validation of Computationally Predicted Substrates for Laccase. Braz. Arch. Biol. Technol. 57, 803-809. doi:10.1590/S1516-8913201402239

Rowell, R. M. (1996). "Physical and Mechanical Properties of Chemically Modified Wood," in Chemical Modification of Lignocellulosic Materials. Editor D. S. Hon (New York, NY: CRC Press), 295-310.

Schubert, M., Ruedin, P., Civardi, C., Richter, M., Hach, A., and Christen, H. (2015). Laccase-catalyzed Surface Modification of Thermo-Mechanical Pulp (TMP) for the Production of wood Fiber Insulation Boards Using Industrial Process Water. PLoS One 10, e0128623. doi:10.1371/journal.pone.0128623

Shanda, J. (2007). Colorimetry: Understanding the CIE Sistem. Hoboken: John Wiley \& Sons.

Slagman, S., Zuilhof, H., and Franssen, M. C. R. (2018). Laccase-mediated Grafting on Biopolymers and Synthetic Polymers: a Critical Review. Chembiochem 19, 288-311. doi:10.1002/cbic.201700518

Sousa, A. C., Piedade, M. F. M. M., Martins, L. O., and Robalo, M. P. (2016). Ecofriendly Synthesis of Indo Dyes Mediated by a Bacterial Laccase. Green. Chem. 18, 6063-6070. doi:10.1039/c6gc02050j

Srinivasulu, C., Ramgopal, M., Ramanjaneyulu, G., Anuradha, C. M., and Suresh Kumar, C. (2018). Syringic Acid (SA) - A Review of its Occurrence, Biosynthesis, Pharmacological and Industrial Importance. Biomed. Pharmacother. 108, 547-557. doi:10.1016/j.biopha.2018.09.069

Su, J., Fu, J., Wang, Q., Silva, C., and Cavaco-Paulo, A. (2018). Laccase: a green Catalyst for the Biosynthesis of Poly-Phenols. Crit. Rev. Biotechnol. 38, 294-307. doi:10.1080/07388551.2017.1354353

Sun, S., Xing, T., and Tang, R. (2014). Enzymatic Dyeing and Functional Finishing of Textile Fibres with Ferulic Acid. Indian J. Fibre Text. Res. 40, 62-69.

Sun, X., Bai, R., Zhang, Y., Wang, Q., Fan, X., Yuan, J., et al. (2013). Laccasecatalyzed Oxidative Polymerization of Phenolic Compounds. Appl. Biochem. Biotechnol. 171, 1673-1680. doi:10.1007/s12010-013-0463-0

Sýs, M., Metelka, R., Frangu, A., Vytřas, K., and Arbneshi, T. (2017). Electrochemical Study of Trametes Versicolor Laccase Compatibility to Different Polyphenolic Substrates. Chemosensors 5, 9. doi:10.3390/chemosensors5010009

Thuring, J. W. J. F., Macdonald, G. J., and Zhuang, W. (2014). Trisubstituted Pyrazoles as Acetylcholine Receptor Modulators. U.S. Patent 8,779,158 B2 (Beerse, BE: United States Patent).

Vidal, L., and Fadli, A. (2007). Composition for Dyeing Keratinous Fibers Comprising at Least One Diamino-N,n-Dihydropyrazolone Derivative. U.S. Patent No. 7,285,137 B2. Paris, FR: United States Patent.
Vitrone, F., Ramos, D., Ferrando, F., and Salvadó, J. (2021). Binderless Fiberboards for Sustainable Construction. Materials, Production Methods and Applications. J. Building Eng. 44, 102625. doi:10.1016/ j.jobe.2021.102625

Xu, F. (1997). Effects of Redox Potential and Hydroxide Inhibition on the $\mathrm{pH}$ Activity Profile of Fungal Laccases. J. Biol. Chem. 272, 924-928. doi:10.1074/ jbc.272.2.924

Yuan, M., Wang, Q., Shen, J., Smith, E., Bai, R., and Fan, X. (2018). Enzymatic Coloration and Finishing of Wool with Laccase and Polyethylenimine. Textile Res. J. 88, 1834-1846. doi:10.1177/0040517517712096

Zerva, A., Simić, S., Topakas, E., and Nikodinovic-Runic, J. (20192009-2019). Applications of Microbial Laccases: Patent Review of the Past Decade (20092019). Catalysts 9, 1023. doi:10.3390/catal9121023

Zhu, T., Liu, S., Ren, K., Chen, J., Lin, J., and Li, J. (2018). Colorability of Dyed wood Veneer Using Natural Dye Extracted from Dalbergia Cochinchinensis with Different Organic Solvents. Bioresources 13, 7197-7211. doi:10.15376/ biores.13.4.7197-7211

Zhuo, R., and Fan, F. (2021). A Comprehensive Insight into the Application of white Rot Fungi and Their Lignocellulolytic Enzymes in the Removal of Organic Pollutants. Sci. Total Environ. 778, 146132. doi:10.1016/j.scitotenv.2021.146132

Conflict of Interest: Authors $\mathrm{AC}$ and $\mathrm{AD}$ were employed by the company BioPox srl.

The remaining author declares that the research was conducted in the absence of any commercial or financial relationships that could be construed as a potential conflict of interest.

Publisher's Note: All claims expressed in this article are solely those of the authors and do not necessarily represent those of their affiliated organizations, or those of the publisher, the editors, and the reviewers. Any product that may be evaluated in this article, or claim that may be made by its manufacturer, is not guaranteed or endorsed by the publisher.

Copyright (c) 2021 Colella, De Chiaro and Lettera. This is an open-access article distributed under the terms of the Creative Commons Attribution License (CC BY). The use, distribution or reproduction in other forums is permitted, provided the original author(s) and the copyright owner(s) are credited and that the original publication in this journal is cited, in accordance with accepted academic practice. No use, distribution or reproduction is permitted which does not comply with these terms. 